\title{
Simultaneous energy harvesting and tribological property improvement
}

\author{
Xiaofan WANG ${ }^{1}$, Jiliang MO ${ }^{1, *}$, Huajiang OUYANG ${ }^{2}$, Zaiyu XIANG ${ }^{1}$, Wei CHEN ${ }^{1}$, Zhongrong ZHOU ${ }^{1}$ \\ ${ }^{1}$ Tribology Research Institute, Southwest Jiaotong University, Chengdu 610031, China \\ ${ }^{2}$ School of Engineering, University of Liverpool, Liverpool L69 3GH, UK \\ Received: 11 May 2020 / Revised: 27 August 2020 / Accepted: 20 October 2020 \\ (C) The author(s) 2020.
}

\begin{abstract}
In this study, piezoelectric elements were added to a reciprocating friction test bench to harvest friction-induced vibration energy. Parameters such as vibration acceleration, noise, and voltage signals of the system were measured and analyzed. The results show that the piezoelectric elements can not only collect vibration energy but also suppress friction-induced vibration noise (FIVN). Additionally, the wear of the friction interface was examined via optical microscopy (OM), scanning electron microscopy (SEM), and white-light interferometry (WLI). The results show that the surface wear state improved because of the reduction of FIVN. In order to analyze the experimental results in detail and explain them reasonably, the experimental phenomena were simulated numerically. Moreover, a simplified two-degree-of-freedom numerical model including the original system and the piezoelectric system was established to qualitatively describe the effects, dynamics, and tribological behaviors of the added piezoelectric elements to the original system.
\end{abstract}

Keywords: piezoelectric; friction-induced vibration (FIV); energy harvester; wear state; contact

\section{Introduction}

Micro-electromechanical systems (MEMS) have developed rapidly in recent years, and low-consumption electronic products such as smart wearable devices, portable electronic devices, and wireless sensors have significantly changed man's everyday life [1]. Most of these products mainly rely on traditional chemical batteries like tiny button batteries which are embedded into the MEMS to provide energy. However, traditional batteries have several disadvantages such as limited lifespan, high labor and production costs caused by the frequent battery replacement, adverse environmental impacts, inconvenient, and sometimes impossible to replace [2-4]. Therefore, seeking an effective method to convert (ambient energy) environmental energy into electric energy has attracted much attention as a research subject in the academic community and industry $[3,5,6]$.
Among the numerous environmental energy sources, environmental vibration energy is abundant and easy to collect. Extensive research has been carried out to study methods in converting environmental vibration energy into electric energy during the past twenty years [3,5]. Four typical conversion methods have been proposed, that is, electromagnetic, piezoelectric, electrostatic, and triboelectric [7-10]. Among them, piezoelectric transduction is shown to be a feasible method to convert vibration energy owing to its simple structure, without the need for an external power supply or magnetic field, and high electromechanical coupling [4, 11-17]. Various developments on this topic have been done by many researchers. Fang et al. [11] designed a nickel-based composite cantilever generator to convert ambient vibration energy into electric energy through piezoelectric effects. Their results show that by adjusting the size of the structure, the generator can undergo resonance in the low-frequency

* Corresponding author: Jiliang MO, E-mail: jlmo@swjtu.cn 
ambient vibration. Moreover, Erturk and Inman [12] proposed an electromechanical model that can be applied to a cantilevered piezoelectric energy harvester that can provide accurate analytical solutions. Sodano et al. [13] showed that the energy generated by the vibration of the piezoelectric materials was sufficient to charge the discharged nickel-metal-hydride (Ni-mh) battery. Also, Yang and Tang [14] built an equivalent circuit model of a piezoelectric energy harvester that could address the challenges of accurate modeling of the electromechanical coupling system, which was demonstrated to have a fair accuracy and show a capability of accounting for the backward coupling effect in piezoelectric energy harvesting system. Additionally, Saadon and Sidek [15], Kim et al. [16], and Erturk and Inman [17] provided systematic reviews on piezoelectric vibration energy harvesting technology.

Although the piezoelectric energy harvesting (PEH) method is able to convert ambient vibration energy into electrical energy with a decent conversion efficiency, this method strongly relies on the vibration property of the ambient environment. In order to maximize the energy conversion efficiency, it is necessary to match the vibration frequency of the external environment with the resonant frequency of the PEH devices [18]. However, the vibration frequency of the external environment is random in most cases and the frequency band of PEH devices is usually limited to a certain range; thus, it is difficult to comprehensively cover the random vibration energy of the external environment, which significantly reduces the conversion efficiency. Therefore, several approaches have been proposed to expand the frequency band of PEH devices so that the vibration frequency of the ambient environment can be covered as much as possible. A study conducted by Liu et al. [4] proposed a generator array based on a thick-film piezoelectric cantilever beam that could improve the frequency band and power output. Moreover, Stewart et al. [19] studied a cantilevered piezoelectric energy harvester and found that the output power of the harvester reached its maximum when the piezoelectric material covered two-thirds of the area. In addition, Yang et al. [20] summarized the methods to broaden the frequency band and highpower output. However, the broadband developments studied above are only suitable for certain applications, and a general solution is not yet available.

Therefore, a new and alternative approach was proposed by various studies to solve this problem, which involves harvesting the vibration energy of friction-induced vibration (FIV). FIV does not rely on the external excitation of the environment vibration source [21-23]. Considering that FIV may cause the generation of unwanted noise (known as frictioninduced vibration and noise (FIVN)), which is often detrimental to people's health and affects operations of machines and functions of structures, a large number of studies on FIV have found potential approaches to reduce it [24-30]. However, it is worth noting that FIV is also beneficial, for example, FIV and other frictional phenomena are associated with acoustics such as the production of the vibration of the strings during the violin and the vibration of the reeds when the accordion is played, and even in energy harvesting [30]. Since FIV energy can be seen in several mechanical applications such as vehicle brakes, mechanical gears, and cutting tools, etc. [31-35], collecting FIV energy through PEH has good prospects. Tsujiura et al. [36] built a self-excited piezoelectric film twin-chip airflow energy harvester and found that a thinner cantilever reduced the maximum output voltage. Tadokoro et al. [37] established an analytical model of a 1-degree-offreedom system with friction and piezoelectric elements, which can be applied to the optimization design of a friction-excited piezoelectric generator oscillator. Several investigations have also verified the feasibility of PEH via stick-slip friction motion using different test setups [38-40]. In a previous work [41] by Wang et al., they studied the influence of different working parameters (external resistance, load, and speed) on PEH by using both experimental and numerical calculation approaches.

Although the above studies have verified the feasibility of PEH via FIV, the effect of FIV energy harvesting on friction and the wear behavior is unclear, and the relationship between the FIV energy harvesting, dynamics, and tribological performance has not been established. Therefore, it is worth carrying out a study to investigate the influence of FIV energy harvesting on the dynamics and tribological behaviors of a friction system, and consequently reveal their relationship. In addition, if part of the vibration energy is transferred 
into electric energy, the noise generated from the friction system may be reduced, which can be considered as a useful and new method to suppress FIVN. These are the main contributions of this work.

In this study, a reciprocating friction test bench was first tested for FIVN, and its energy was then collected. The vibration and noise signals in the original state and energy collection state were recorded and compared to study the influence of the FIV energy collection on the friction system. MATLAB and ABAQUS 6.14 software were used to conduct a detailed analysis of the experimental results by means of numerical calculations. Several useful findings are given in the end.

\section{Details of the test process}

\subsection{Introduction of test apparatus}

The test was carried out on a reciprocating friction and wear test bench. The schematic diagram of the apparatus is shown in Fig. 1. The test apparatus is mainly composed of a signal processing system, a ball-on-disc contact system, and a bracket system. A biaxial force sensor was mounted on the top of the test apparatus, which was able to measure force in normal and tangential directions. A suspension was installed at the bottom of the force sensor through threaded screws that were used to protect the force sensor from abnormal overload and maintain a relatively constant normal load between the contact surfaces. To measure the vibration in real-time, a triaxial acceleration sensor was installed on the ball holder. The ball sample (chromium bearing steel ball, $D=$ $10 \mathrm{~mm}, E=210 \mathrm{GPa}$, AISI 52100, $\mathrm{HV}_{0.05}=510 \mathrm{~kg} / \mathrm{mm}^{2}$, and $\sim 0.02 \mathrm{~mm} \mathrm{Ra}$ ) used in the test was fixed by a ball clamp and was in contact with the lower disc sample. The disc sample used in the test (cast iron with a radius of $12.5 \mathrm{~mm}$ and a thickness of $3 \mathrm{~mm}$ ) was adhered to the sliding table, which was driven by the reciprocating sliding device to achieve reciprocating motion. During the test, the microphone was placed in a horizontal position with the contact interface at a distance of $10 \mathrm{~cm}$ to measure the noise signal. In the entire testing process, all sensors were connected to the signal processing system, and synchronous measurement

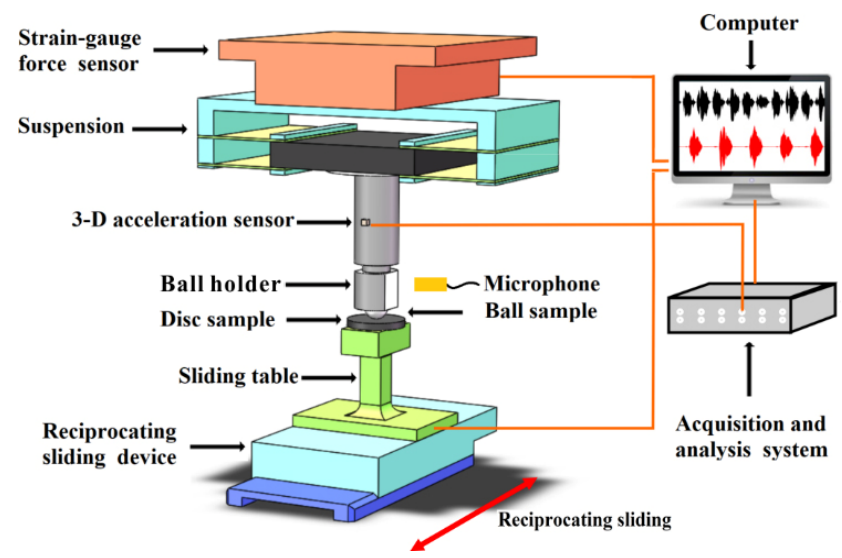

Fig. 1 Schematic diagram of reciprocating friction and wear test bench.

and processing of force, vibration, and noise signals were realized.

\subsection{Introduction of the piezoelectric elements of the test apparatus}

Considering that FIV was generated in both the normal and tangential directions simultaneously, two cantilever beams (size: $60 \mathrm{~mm} \times 12 \mathrm{~mm} \times 0.1 \mathrm{~mm}$, material: 304 stainless steel) were installed on the ball holder, which were used to absorb the vibration energies in the two directions normal to their respective surfaces. It is worth noting that the position of the cantilever beam should be chosen reasonably considering the ball holder's structural properties. In this work, the ball holder has a long bar structure; thus, the vibration levels at different positions of the holder show a significant difference, and the vibration modes of this friction system should be computed to estimate the likely largest deformation position in this ball holder. This work was performed in the authors' previously published work [42], and the largest deformation position was found to be at the bottom part of the ball holder.

A schematic diagram of the ball holder with cantilever beams is shown in Fig. 2(a). A piezoelectric film (PVDF: $50 \mathrm{~mm} \times 10 \mathrm{~mm} \times 0.03 \mathrm{~mm}$ ) was adhered on one side of the surface of the cantilever beam. The positive and negative electrodes of the piezoelectric film were connected through wires with a load resistance $(R=10 \mathrm{k} \Omega)$ in the interface circuit. Both the normal and tangential piezoelectric voltage signals generated during the friction process can be detected 
(a)

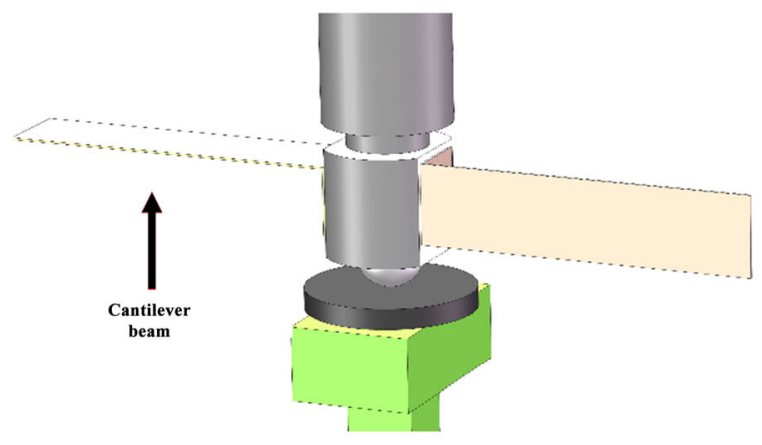

(b)

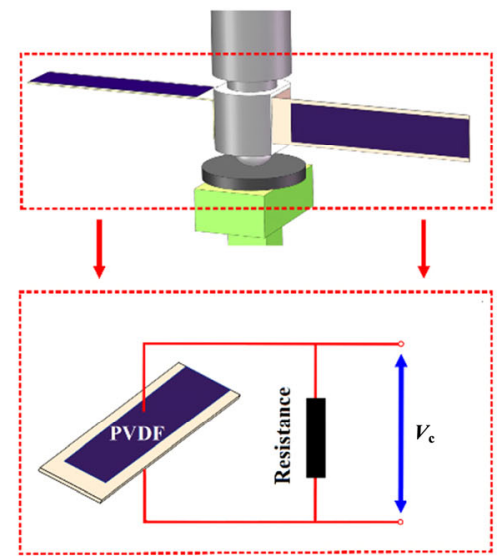

Fig. 2 Schematic diagrams of the two different systems: (a) ball holder with cantilever beams (cantilever system), and (b) with piezoelectric elements (piezoelectric system) and circuit.

and recorded synchronously via the signal processing system. A schematic diagram of the test apparatus and circuit is shown in Fig. 2(b). For convenience, the reciprocating friction and wear test system without the cantilevers is called the original system, while the system with them is called the cantilever system; the system with them and the piezoelectric elements is called the piezoelectric system.

Before performing the tests, the disc sample surface was polished using sandpapers and a polishing cloth, and the surfaces of the ball sample and the disc sample were swabbed with ethanol to prevent contaminants from affecting the contact interface. For the test parameters, the normal load was set at $10 \mathrm{~N}$, reciprocating sliding displacement was fixed at $4 \mathrm{~mm}$, the sliding frequency was $1 \mathrm{~Hz}$, and the testing time was $1,800 \mathrm{~s}$. The tests were carried out under strict environmental conditions $\left(25-27^{\circ} \mathrm{C}\right.$ and $\left.60 \pm 5 \% \mathrm{RH}\right)$. Each test was repeated more than 4 times to ensure the reliability of the results.

\section{Test results and signal analysis}

\subsection{Coherence analysis}

In order to verify that the PEH is feasible via FIV, a correlation analysis between the output voltage and vibration acceleration signals was performed. Figure 3(a) illustrates the output voltage and vibration acceleration signals of the piezoelectric system at a sampling frequency of $20 \mathrm{kHz}$ in the normal and tangential directions. It can be seen that the vibration signals in each direction exhibit large amplitudes during this period $(1,400-1,405 \mathrm{~s})$, which indicates that the FIV is generated in these two directions. In addition, the normal and tangential output voltage signals show obvious fluctuations after the piezoelectric elements are added into the friction system. This phenomenon means that the piezoelectric elements respond to vibrations and convert vibration energy into electrical energy.

Figure 3(b) shows the frequency spectra of the output voltage and vibration acceleration signals through a fast Fourier transform (FFT). Visibly, both frequency spectra display a major frequency of approximately $910 \mathrm{~Hz}$. Accordingly, the correlation between the acceleration and the voltage was studied through a coherence analysis, as shown in Fig. 3(c). The figure shows that the coherence coefficient values are mostly greater than 0.7 , in the range of 0 to $4,000 \mathrm{~Hz}$, and the coherence coefficient value is close to 1 near $900 \mathrm{~Hz}$; thus, it can be concluded that the output voltage signal and vibration signal have good coherence. It is proved that part of the kinetic energy induced by the friction is converted into electricity by using piezoelectric elements.

\subsection{Noise and vibration analysis of friction systems}

\subsubsection{Noise evolution analysis}

Figure 4 shows the root mean square (RMS) values of noise signals generated by the different systems during the test, which was used to study the influence of piezoelectric elements and PEH on noise generation. 

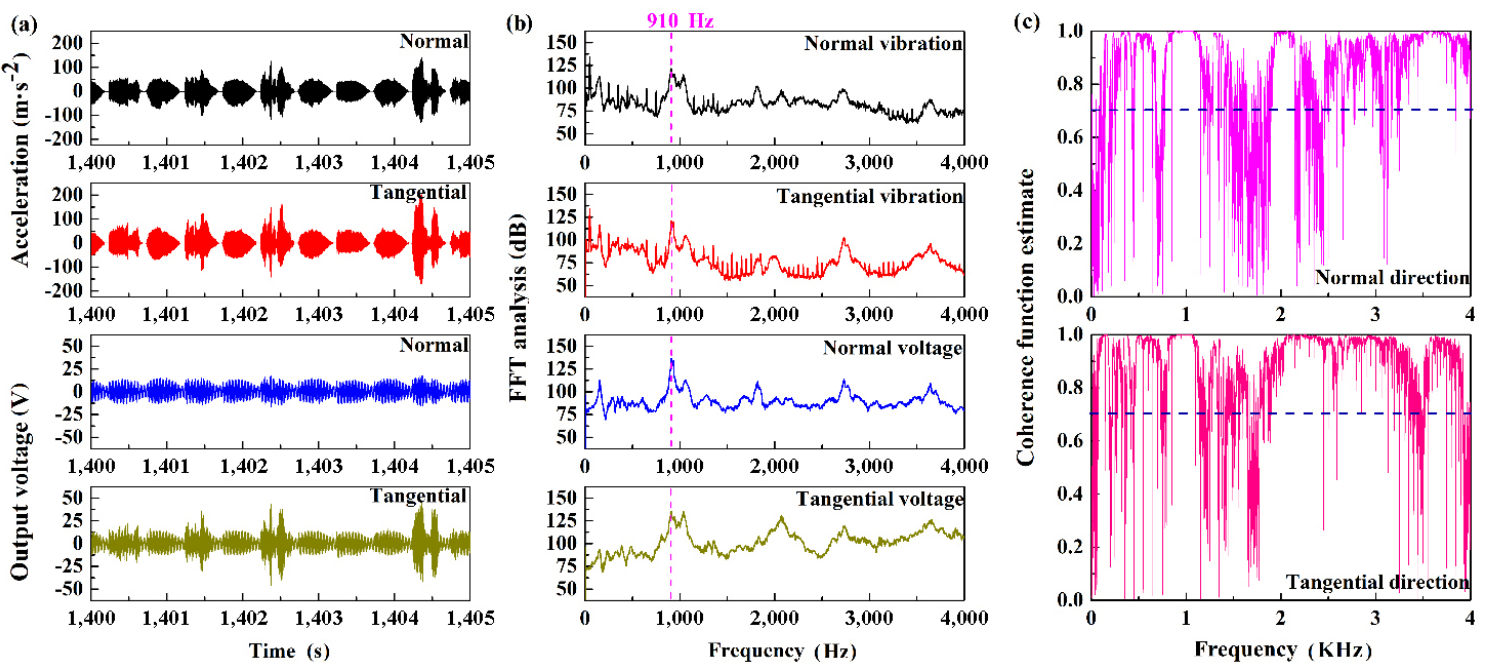

Fig. 3 Vibration accelerations signals of the friction-piezoelectric system in the normal and tangential directions and the corresponding voltage signals (a); frequency spectra of the output voltage and the vibration acceleration signals (b); and results of the coherence analysis (c).

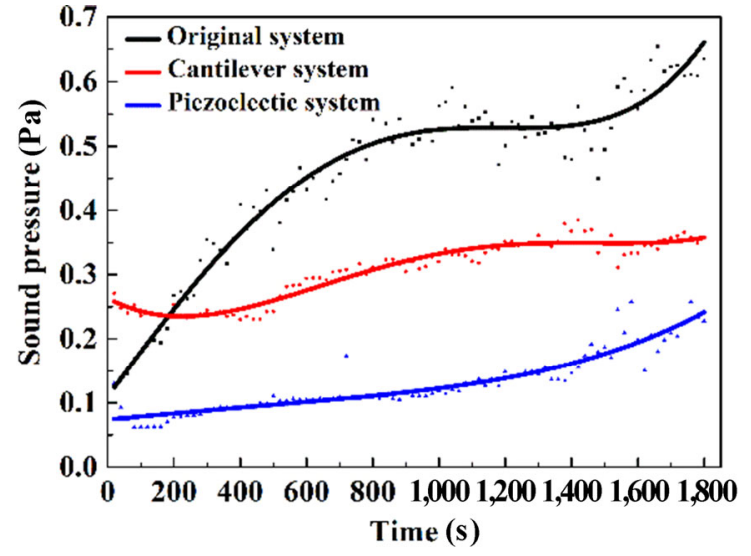

Fig. 4 Fitted curves of RMS values of sound pressures generated by three friction systems.

It is found that the sound pressure of the original friction system shows a relatively low level at the beginning, and then it tends to increase rapidly with time. For the cantilever and piezoelectric systems, the growth trends of the sound pressure curves remain relatively steady during the entire process. The results show that the sound pressure generated from the piezoelectric system is at the lowest level among all three systems. Therefore, the piezoelectric system is capable of reducing the sound pressure level of the friction system.

\subsubsection{Vibration analysis in different friction stages}

In this work, the analysis of the entire experimental process was divided into three stages: The initial stage
(300-305 s), the middle stage (900-905 s), and the final stage (1,400-1,405 s). The vibration signals of the three systems in three different stages are shown in Fig. 5 to further verify the capability of the piezoelectric elements in improving the FIV behavior.

During the initial stage, the normal vibration acceleration signal of the original system shows a large amplitude, while for both the cantilever and piezoelectric systems, the vibration acceleration signals are weaker, and the vibration amplitude of the piezoelectric system is the lowest among these three systems. In the middle stage, the vibration acceleration signals of both the original system and cantilever system show a significant increase, while the vibration signal generated from the piezoelectric system remains at a low level. In the final stage, although the vibration acceleration signals of the three friction systems exhibit further increases, the vibration level of the piezoelectric system is still the lowest among the three. In summary, the vibration level of the piezoelectric system is the lowest, which indicates that the addition of piezoelectric elements in the friction system can effectively reduce the FIV intensity.

\subsubsection{Time-frequency analysis of the vibration signals in different friction stages}

Figure 6 shows the frequency spectrum analysis of the vibration acceleration signals of different systems in the time periods of 300-305 s, 900-905 s, and 

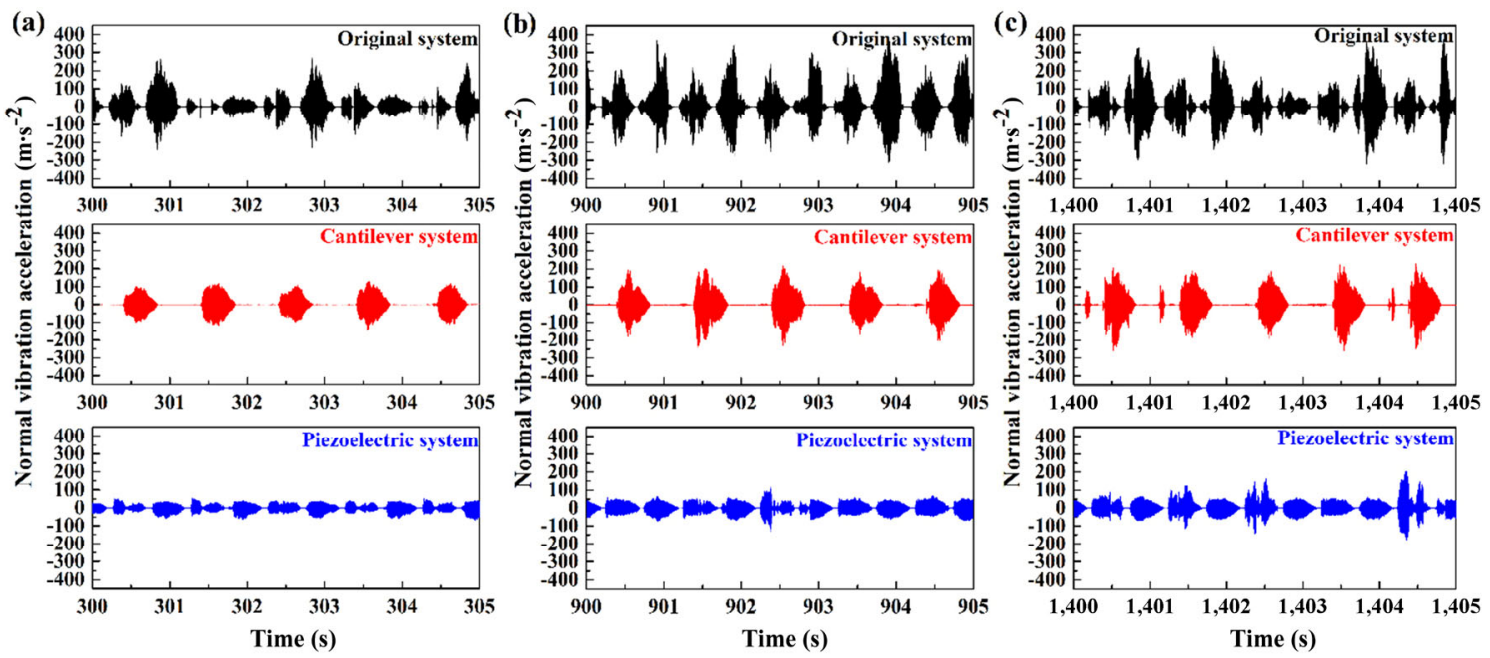

Fig. 5 Time-domain diagram of normal vibration acceleration signals of different systems in the time periods: (a) 300-305 s; (b) 900-905 s; and (c) 1,400-1,405 s.

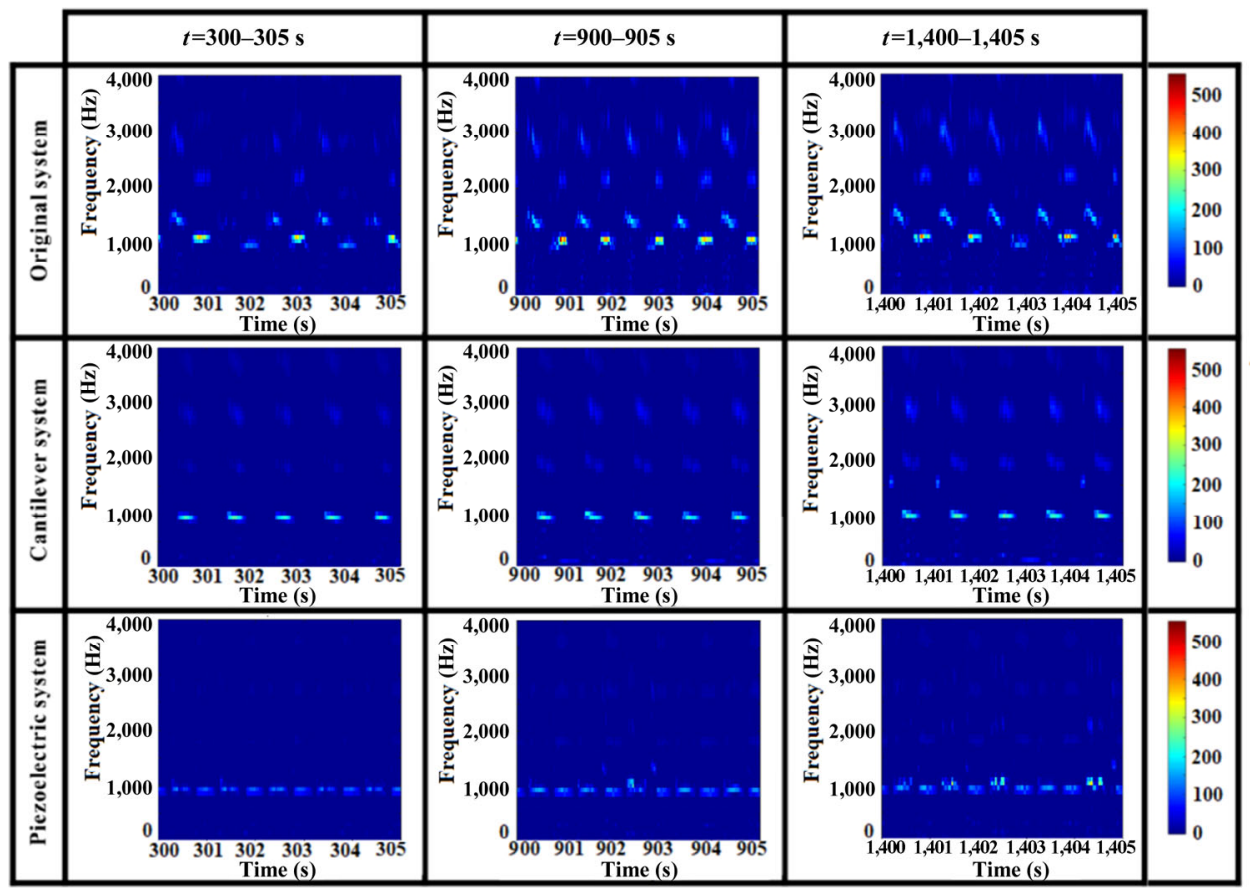

Fig. 6 Frequency spectrum analysis diagram of the normal vibration acceleration signals.

$1,400-1,405 \mathrm{~s}$. The main frequency of $1,060 \mathrm{~Hz}$ could be found in the original friction system, which indicates that the original system tends to generate squeal noise. Moreover, several super harmonics could also be identified, and the noise characteristics are rather complex, which may account for the complicated tribological behavior at the contact interface. At the same time, similar main frequencies with relatively low energy could be detected at different stages in the cantilever system, indicating that the cantilever system may generate squeal instability, but at a lower level.

For the piezoelectric system, only one main frequency at a low energy level was detected in three different stages, which further demonstrates that the addition of piezoelectric elements can effectively reduce the FIV level. The reason may be that the tribological behavior on the interface is effectively improved, and consequently, the wear on the friction interface is alleviated after introducing the cantilever beam and 
the piezoelectric elements, which in turn helps to suppress the FIVN and simplify its frequency content.

\subsection{System friction and wear analysis}

\subsubsection{Friction force analysis}

Figure 7(a) illustrates the friction force curves recorded in the steady stage. Clearly, the friction force of the original system shows the largest amplitude (approximately $4 \mathrm{~N}$ ) compared with the cantilever and piezoelectric systems, and the piezoelectric system exhibited the lowest amplitude of friction force (close to $3 \mathrm{~N}$ ) among the three friction systems. In order to compare the frictional force of the three systems more intuitively, the RMS values of the frictional force were calculated during the entire test process. As shown in Fig. 7(b), the RMS value of the original system was $4.73 \mathrm{~N}$, while the value of the piezoelectric system was $4.21 \mathrm{~N}$. Therefore, adding a cantilever beam and piezoelectric elements into the friction system is

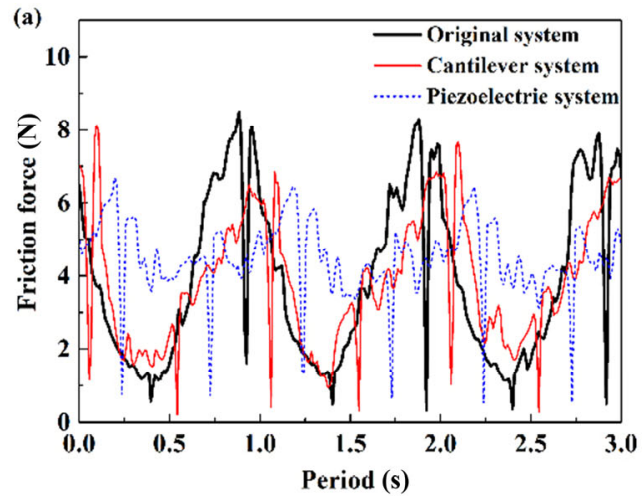

able to make the friction force more stable, that is, it underlies a smaller temporal fluctuation.

\subsubsection{Wear topography analysis}

In order to explore the influence of the addition of piezoelectric elements into the friction system on the wear behavior, the worn surface morphologies of the disc and ball samples after the tests were examined using optical microscopy (OM). The aim of this procedure was to establish the relationship between the $\mathrm{PEH}$, noise, and vibration performance, and friction and wear. Figure 8 shows the worn surface morphologies of the ball and disc samples of the different friction systems. For the original system, the contact surface of the disc and the ball samples exhibited a large worn area and visible ploughing, with noticeable debris accumulation and surface damage observed in the wear track. In contrast, the contact surfaces of the disc and the ball samples of the cantilever system and piezoelectric system show

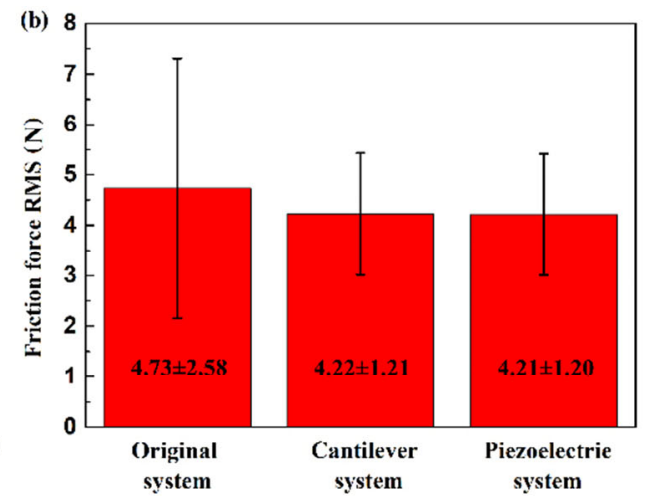

Fig. 7 Result of the friction force analysis. (a) Friction force curves recorded in three cycles of steady stage and (b) RMS analysis of the whole process.

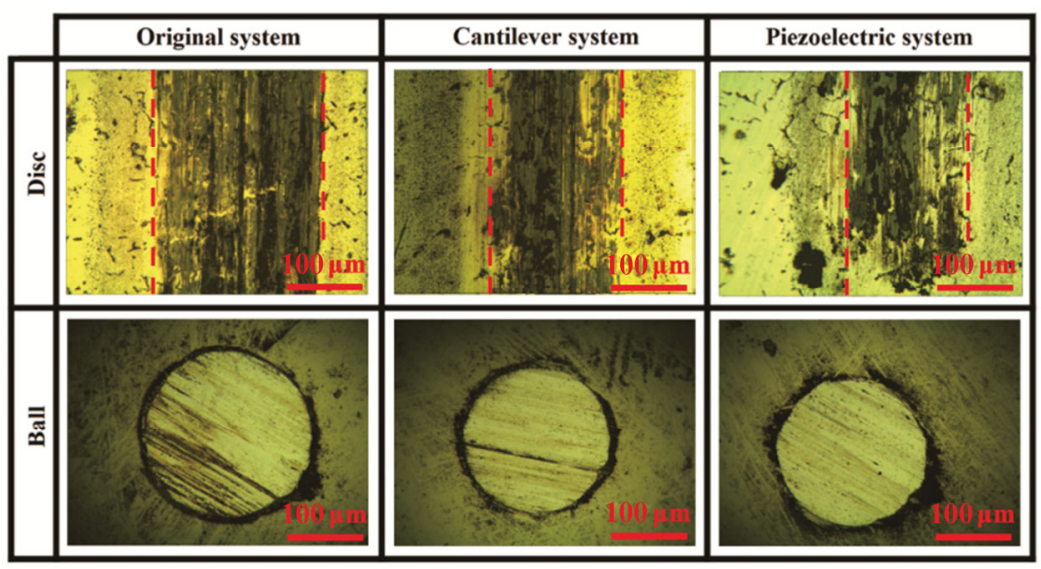

Fig. 8 OM images of the disc and ball sample surfaces of three friction systems. 
relatively mild wear, especially for the piezoelectric system. The sample surfaces have the smallest wear area.

Accordingly, white-light interferometry (WLI) analysis was performed to further examine the wear state of the disc sample surfaces as shown in Fig. 9(a). Meanwhile, the wear scar profiles of disc samples of the three friction systems are illustrated to analyze the differences between them, which is shown in Fig. 9(b). It was found that the sample surfaces of the original system exhibited more severe wear and deeper wear scar profiles with a maximum wear depth of approximately $3.5 \mu \mathrm{m}$. The sample surface of the piezoelectric system shows very mild wear and very shallow wear scar profiles with a maximum wear depth of approximately $1.3 \mu \mathrm{m}$ compared with the other two systems.

It is difficult to calculate the wear area of the ball samples precisely because the surface wear area is approximately circular. In order to compare and analyze the surface of ball samples of the three kinds of friction systems, it is simplified as a circle in the calculation. Figure 9(c) shows the wear areas of the friction surfaces of the ball samples of the three friction systems. It can be clearly seen that the original system has the largest wear area (approximately $0.457 \mathrm{~mm}^{2}$ ), while the piezoelectric system has the smallest wear area (approximately $0.306 \mathrm{~mm}^{2}$ ). This conclusion is consistent with the conclusion of Fig. 8.

Furthermore, scanning electron microscopy (SEM) was used to observe the wear morphology as presented in Fig. 10. For the original system, the wear area of the disc sample is very large, and the surface exhibits severe and complicated wear behavior. A visible material exfoliation can be observed in the wear track. In order to further study the specific conditions of the wear scar surface, three points were taken on the wear scar surface of the original system for energy dispersive X-ray spectroscopy (EDX) analysis. The surface wear debris was removed by air blowing in advance to prevent the scattered abrasive debris from interfering with the experiment. The results are shown in Fig. 11. Figure 11(a) shows the EDX analysis of point A, wherein a large amount of Fe elements and a small amount of Si elements exist, indicating that the material around point $\mathrm{A}$ is the matrix material that has not been worn out in the disc sample. Point B is located on a small bump. The result of Fig. 11(b) shows that
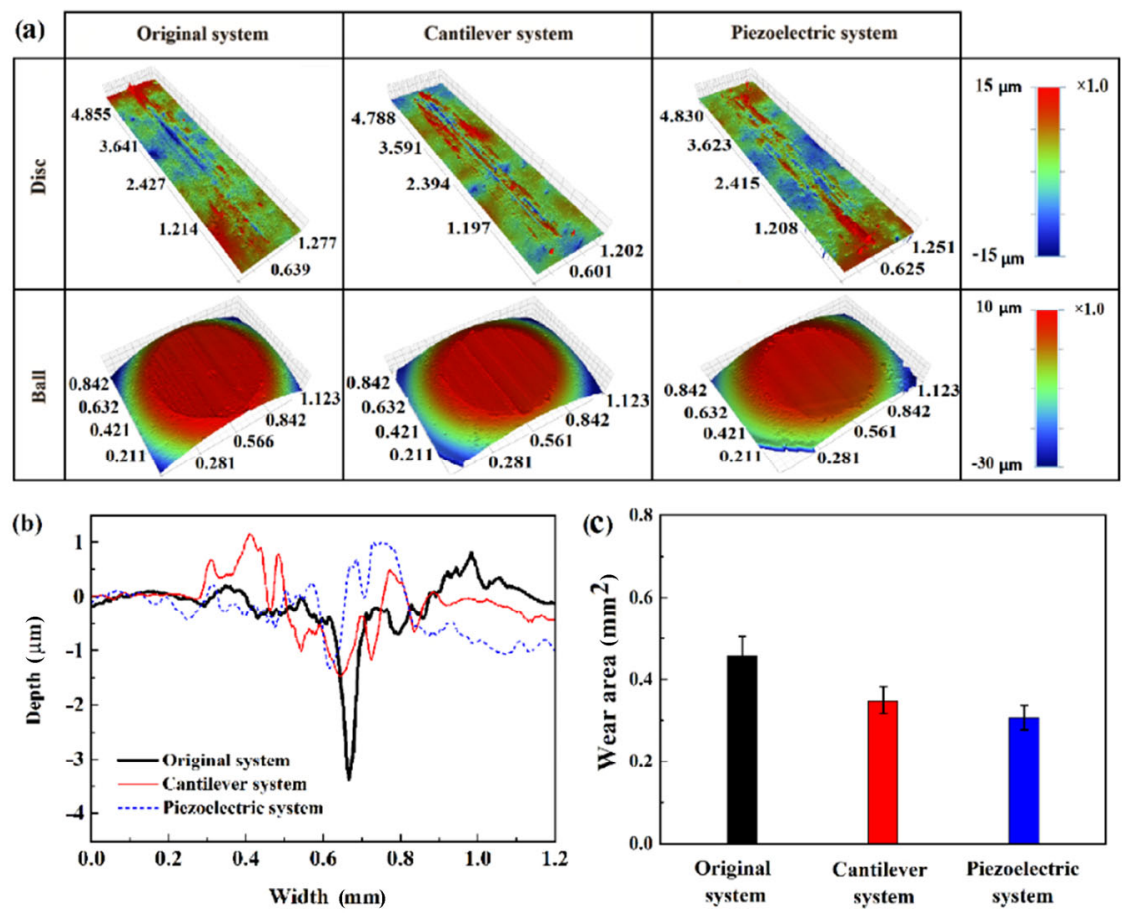

Fig. 9 Results of the wear topographies analysis. (a) 3D surface topographies of the disc and ball sample surfaces of three friction systems, (b) wear scar profiles of disc sample of three friction systems, and (c) wear areas of the friction surface of the ball sample of the three friction systems. 

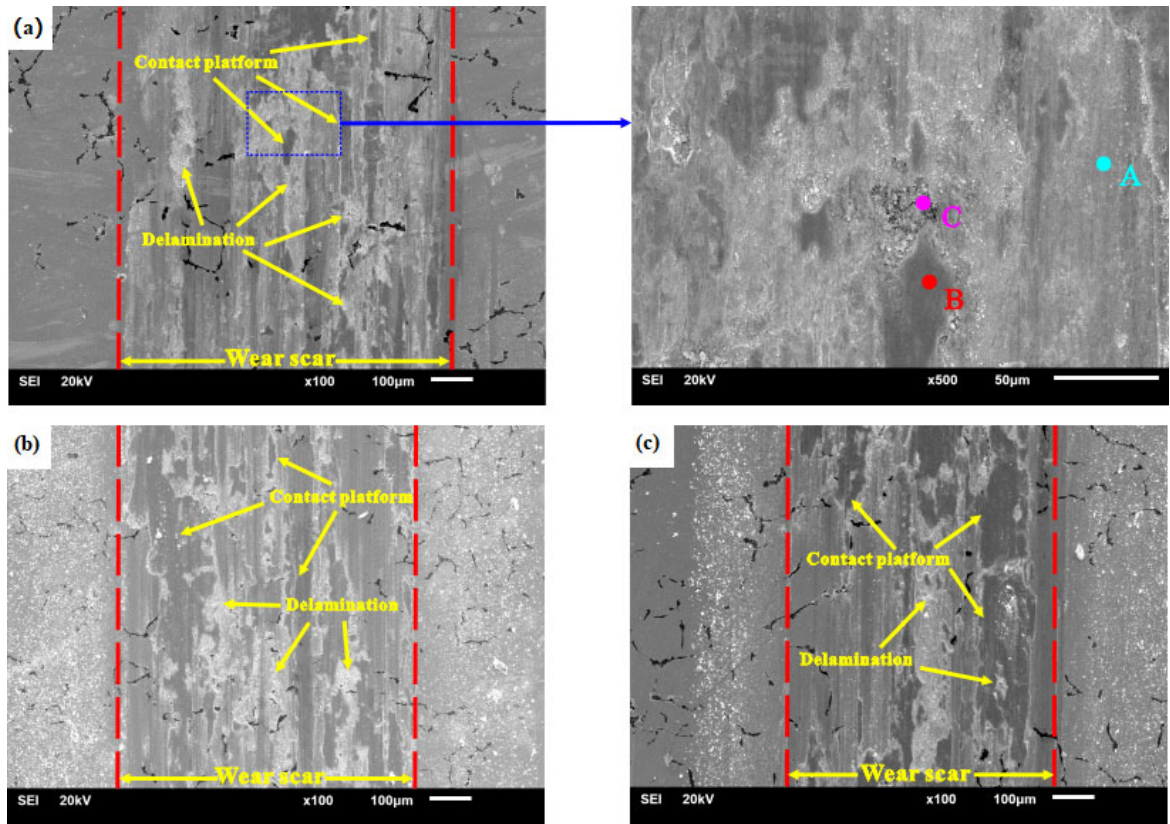

Fig. 10 SEM images for the three systems. (a) The disc sample surfaces of the original system, (b) the cantilever system, and (c) the piezoelectric system.

(a)

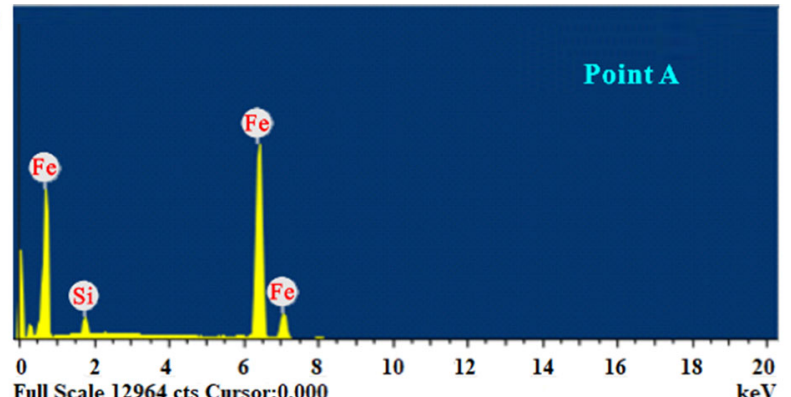

(b)

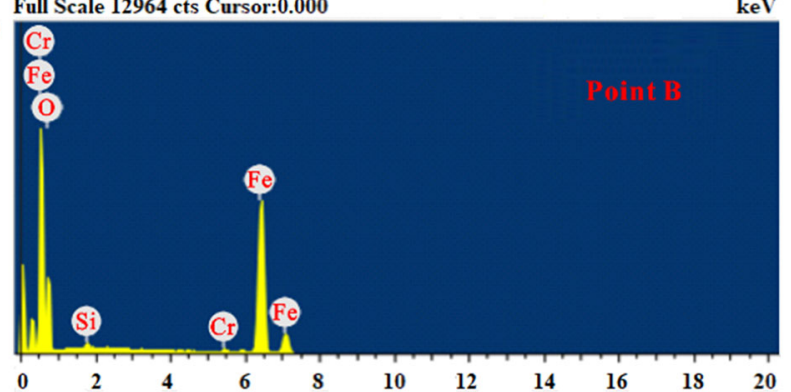

(c)

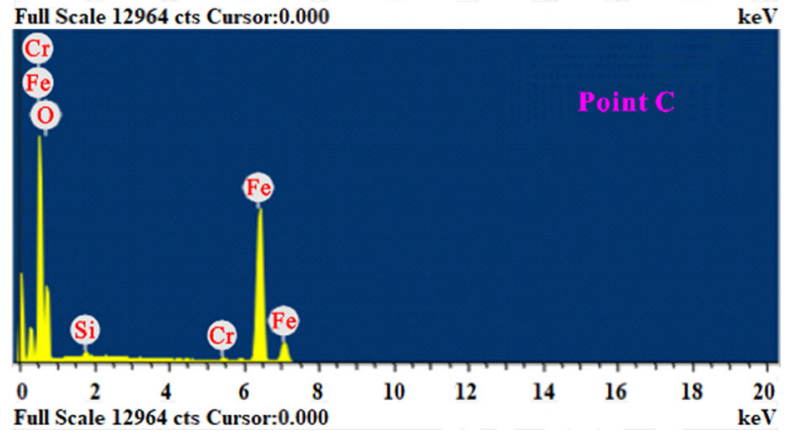

Fig. 11 EDX analysis of three points on the worn surface of the original system. point $\mathrm{B}$ has a large number of $\mathrm{O}$ and $\mathrm{Cr}$ elements in addition to the Fe element. The appearance of the $\mathrm{O}$ element indicates the occurrence of oxidative wear, and the $\mathrm{Cr}$ element is only possessed by the ball sample, which proves that a large amount of debris is accumulated and compacted to form contact plateaus after repeated friction. The EDX analysis of point $C$ is shown in Fig. 11(c). The spectrum of point $C$ is almost the same as that of point $\mathrm{A}$, and there are several scattered contact plateaus around it, which provides clear evidence that the contact plateaus evolve from the accumulation of abrasive debris.

Figure 10(b) shows that a large number of scattered contact plateaus and material spalling appear on the wear surface of the cantilever system. Compared with the other two systems, the disc sample of the piezoelectric system exhibits the lowest wear level, as shown in Fig. 10(c). The piezoelectric system shows some unworn areas and a relatively complete contact plateau. Although material micro-spalling still appears on the worn surface, it is much milder than the situations of the original system and the cantilever system. Therefore, linking the noise and vibration results shown in Figs. 4 and 5, it can be concluded that the more severe wear surface has a greater tendency to promote the generation of unstable vibration and higher-level. The addition of piezoelectric elements 
can effectively reduce the wear level of the contact surfaces and improve the FIVN performance.

\section{Numerical analysis}

\subsection{The finite element model and corresponding load and boundary conditions of the friction system}

In this section, the finite element model of the friction system was built according to the actual geometric size of the components by using ABAQUS 6.14 software, which is shown in Fig. 12(a). This model used in the study was composed of eight components of the test system: strain-gauge force sensor, suspension, ball holder, two cantilever beams, ball sample, disc sample, sliding table, and reciprocating sliding device.

The corresponding load and boundary conditions are shown in Fig. 12(b). A normal load of $20 \mathrm{~N}$ was applied on the top surface of the force sensor, which is constrained in all degrees of freedom except for the translation degree of freedom in the Z-direction. The bottom of the reciprocating sliding device was fixed in the $Y$ and $Z$ directions, and a sinusoidal velocity boundary condition was applied in the $X$-direction. The contact between the ball sample and the disc is defined as frictional contact using the Coulomb friction law in the numerical model. The surface of the disc is defined as the master surface because of its coarser mesh than that of the pad. The friction coefficient was set at 0.4 , which represents the average value obtained from the experiment. The other parts are always in
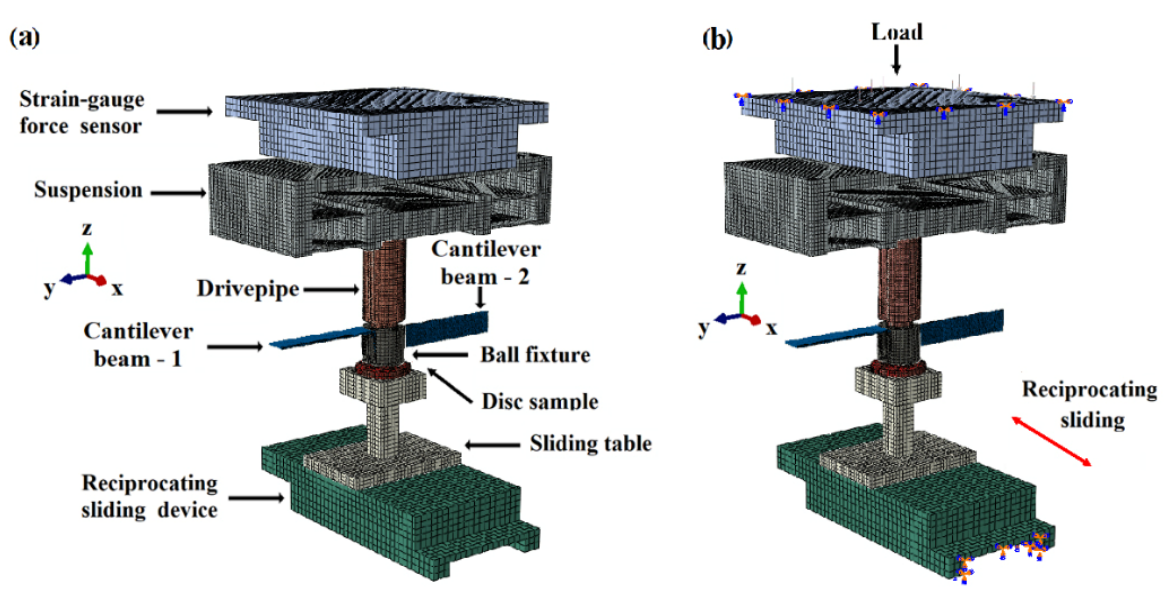

Fig. 12 Diagram of the model used for the numerical analysis. (a) The finite element model of the cantilever system and (b) corresponding load and boundary conditions. into the friction system is able to achieve energy conversion and improve the surface wear state simultaneously, a simplified mathematical model was established to qualitatively explain the above-mentioned experimental observations [43]. The two-degree-offreedom numerical model proposed in this section is mainly used to simulate the basic characteristics of the original system and the piezoelectric system, in order to compare the differences between the tribological behaviors of the two systems. It can also prove the feasibility of the piezoelectric system for converting vibration energy. The results of numerical simulations are not meant to reproduce the experimental results in a quantitative manner; thus, the parameters of the mathematical model are arbitrarily set. Even though the parameter values are not really those of

close contact with each other, and the contact state has little effect on the calculation; therefore, a tie constraint obtain high computational efficiency.

The ABAQUS/standard solver is used to calculate the deformation of the cantilever beams during the friction process, as shown in Fig. 13. It can be seen that the two cantilever beams show a visible displacement variation in the normal and tangential directions, during the friction process, and the vibration energy is transferred into electric energy if the piezoelectric elements are added.

\subsection{The two-degree-of-freedom numerical model}




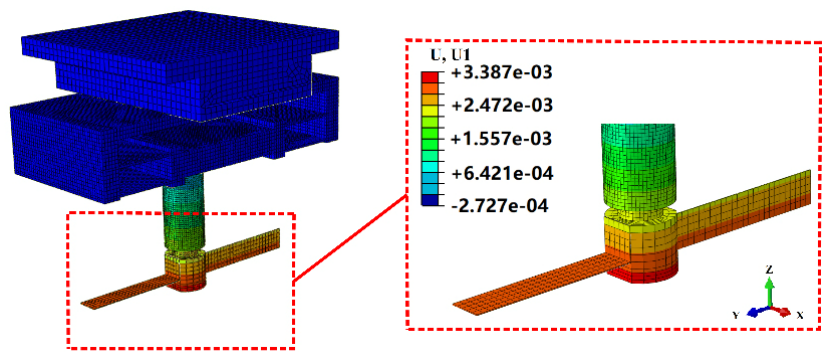

Fig. 13 The displacement cloud diagram of the cantilever beam.

the actual system, the theoretical results should reflect how the friction systems should behave.

The model of the original friction system is shown in Fig. 14(a). A mass (M), representing the ball sample, is connected with springs $k_{1}$ and $k_{2}$. A normal load $F$ pushes the mass to come into contact with a rigid belt, representing the disc, moving at a constant speed. The speed of the belt was $V$. The coefficient of friction at the sliding interface is denoted by $\mu$, and the stick-slip vibration is assumed not to occur. The normal contact stiffness between the belt and the mass is expressed by springs $k_{3}$ and $k_{4}$, where $k_{1}, k_{2}$, and $k_{3}$ are all linear springs and $k_{4}$ is a nonlinear cubic spring. This model is identical to the one studied in Ref. [43], except that the current model does not have horizontal and vertical dampers or piezoelectric elements.

The motion equation matrix of the original system is [43]:

$$
\begin{aligned}
& {\left[\begin{array}{cc}
M & 0 \\
0 & M
\end{array}\right]\left\{\begin{array}{l}
\ddot{\boldsymbol{x}} \\
\ddot{y}
\end{array}\right\}+\left[\begin{array}{cc}
\frac{\boldsymbol{k}_{1}}{2}+\boldsymbol{k}_{2} & -\frac{\boldsymbol{k}_{1}}{2}+\mu \boldsymbol{k}_{3} \\
-\frac{\boldsymbol{k}_{1}}{2} & \frac{\boldsymbol{k}_{1}}{2}+\boldsymbol{k}_{3}
\end{array}\right]\left\{\begin{array}{l}
\boldsymbol{x} \\
y
\end{array}\right\}} \\
& +\left[\begin{array}{cc}
0 & \boldsymbol{\mu} \boldsymbol{k}_{4} \\
0 & \boldsymbol{k}_{4}
\end{array}\right]\left\{\begin{array}{c}
\boldsymbol{x}^{3} \\
\boldsymbol{y}^{3}
\end{array}\right\}=\left\{\begin{array}{c}
0 \\
-\boldsymbol{F}
\end{array}\right\}
\end{aligned}
$$

(a)

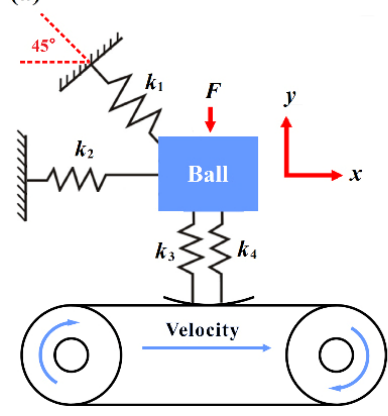

(b)

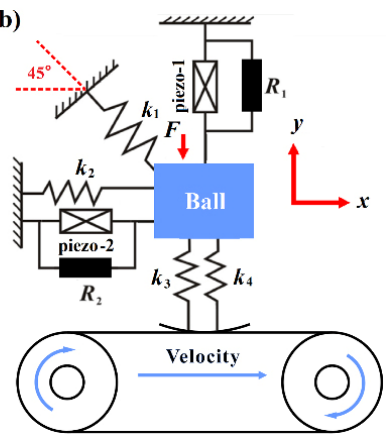

Fig. 14 The simplified two-degree-of-freedom numerical models for (a) original system and (b) piezoelectric system.
The simplified two-degree-of-freedom model of the piezoelectric system is shown in Fig. 14(b), based on that of the original system presented in Fig. 14(a). Piezo-1 and Piezo-2 are piezoelectric elements connected with mass in the vertical and horizontal directions, respectively. The equations of the piezoelectric elements linking the electrical variables and the mechanical variables are as follows $[39,44]$ :

$$
\begin{aligned}
& \frac{U_{1}}{R_{1}}=\alpha_{1} \dot{y}-C_{1} \dot{U}_{1} \\
& \frac{U_{2}}{R_{2}}=\alpha_{2} \dot{x}-C_{2} \dot{U}_{2}
\end{aligned}
$$

where $U_{1}$ and $U_{2}, C_{1}$ and $C_{2}$, and $\alpha_{1}$ and $\alpha_{2}$ are the output voltages, clamped capacitance, and force factors of piezoelectric elements of Piezo-1 and Piezo-2, respectively.

It was assumed that mass $M$ does not separate from the belt during vibration. Thus, the motion equation matrix of the piezoelectric friction system is:

$$
\begin{aligned}
& {\left[\begin{array}{cccc}
\boldsymbol{M} & 0 & 0 & 0 \\
0 & \boldsymbol{M} & 0 & 0 \\
0 & 0 & 0 & 0 \\
0 & 0 & 0 & 0
\end{array}\right]\left\{\begin{array}{c}
\ddot{\boldsymbol{x}} \\
\ddot{\boldsymbol{y}} \\
\ddot{\boldsymbol{U}}_{1} \\
\ddot{\boldsymbol{U}}_{2}
\end{array}\right\}+\left[\begin{array}{cccc}
0 & 0 & 0 & 0 \\
0 & 0 & 0 & 0 \\
\boldsymbol{\alpha}_{2} & 0 & 0 & -\boldsymbol{C}_{2} \\
0 & \boldsymbol{\alpha}_{1} & -\boldsymbol{C}_{1} & 0
\end{array}\right]\left[\begin{array}{c}
\dot{\boldsymbol{x}} \\
\dot{\boldsymbol{y}} \\
\dot{\boldsymbol{U}}_{1} \\
\dot{\boldsymbol{U}}_{2}
\end{array}\right\}} \\
& +\left[\begin{array}{cccc}
\frac{\boldsymbol{k}_{1}}{2}+\boldsymbol{k}_{2} & -\frac{\boldsymbol{k}_{1}}{2}+\boldsymbol{\mu} \boldsymbol{k}_{3} & 0 & \boldsymbol{\alpha}_{2} \\
-\frac{\boldsymbol{k}_{1}}{2} & \frac{\boldsymbol{k}_{1}}{2}+\boldsymbol{k}_{3} & \boldsymbol{\alpha}_{1} & 0 \\
0 & 0 & 0 & -\frac{1}{\boldsymbol{R}_{2}} \\
0 & 0 & -\frac{1}{\boldsymbol{R}_{1}} & 0
\end{array}\right]\left\{\begin{array}{c}
\boldsymbol{x} \\
\boldsymbol{y} \\
\boldsymbol{U}_{1} \\
\boldsymbol{U}_{2}
\end{array}\right\} \\
& +\left[\begin{array}{cccc}
0 & \boldsymbol{\mu} \boldsymbol{k}_{4} & 0 & 0 \\
0 & \boldsymbol{k}_{4} & 0 & 0 \\
0 & 0 & 0 & 0 \\
0 & 0 & 0 & 0
\end{array}\right]\left\{\begin{array}{c}
\boldsymbol{x}^{3} \\
\boldsymbol{y}^{3} \\
\boldsymbol{U}_{1}^{3} \\
\boldsymbol{U}_{2}^{3}
\end{array}\right\}=\left\{\begin{array}{c}
0 \\
-\boldsymbol{F} \\
0 \\
0
\end{array}\right\}
\end{aligned}
$$

The following parameter values were used in the numerical analysis: $M=5 \mathrm{~kg}$, the normal load $F=20 \mathrm{~N}$, the speed of the belt $V=0.1 \mathrm{~m} / \mathrm{s}$, the stiffness of three linear springs are $k_{1}=60 \mathrm{~N} / \mathrm{m}, k_{2}=100 \mathrm{~N} / \mathrm{m}, k_{3}=50 \mathrm{~N} / \mathrm{m}$, and stiffness of the nonlinear cubic spring $k_{4}=300 \mathrm{~N} / \mathrm{m}^{3}$. The coefficients of kinetic friction $\mu_{\mathrm{k}}$ and the coefficient of static friction $\mu_{\mathrm{s}}$ are kept to a constant value of 0.4 and 0.5 , respectively. The critical coefficient of friction was found to be 0.33 . The clamped capacitance of 
piezoelectric elements $C_{1}=C_{2}=7.2 \times 10^{-6} \mathrm{~F}$, the force factors of piezoelectric elements $\alpha_{1}=\alpha_{2}=0.015 \mathrm{~N} / \mathrm{V}$, and the resistance $R_{1}=R_{2}=10 \mathrm{k} \Omega$.

The $3 \mathrm{D}$ phase diagrams of output voltages and velocities varying with time of the piezoelectric system in both the vertical and horizontal directions are shown in Fig. 15. It was found that the output voltage and velocity signals show a certain level of oscillation with time, and both of these signals exhibit visible stable limit cycles, which indicates that the FIV energy is transferred into electric energy. Figure 16 shows the time-domain output voltages of the piezoelectric system in two directions. It was found that the output voltages in the normal and tangential directions show large oscillations, which further indicates that the piezoelectric system could convert the vibration energy into electric energy.

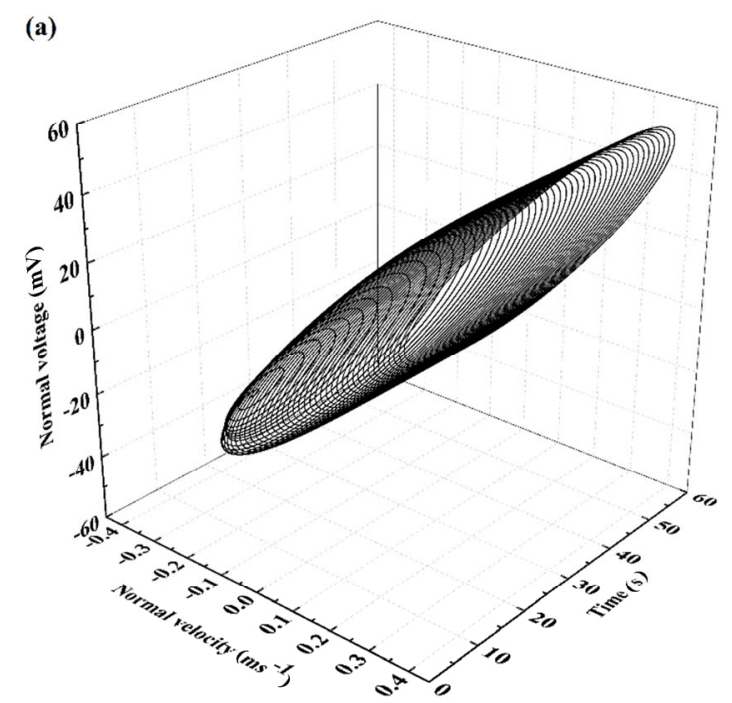

\subsection{Comparison and analysis of original system and piezoelectric system}

The time histories of the displacement of the original system and the piezoelectric system in the normal and tangential directions over time within $60 \mathrm{~s}$ were calculated and were shown in Fig. 17. It can be clearly seen that the displacement amplitude of the piezoelectric system is smaller than that of the original system, and the displacement amplitude becomes steady more quickly. Figure 18 shows the phase plots and Poincare maps of the original system and the piezoelectric system. It was found that for both systems, the trajectories of the phase diagrams are close to a circle in the normal direction. The upper part of the trajectories remained horizontal, showing a stick-slip phenomenon in the tangential direction. Figures 18(a)

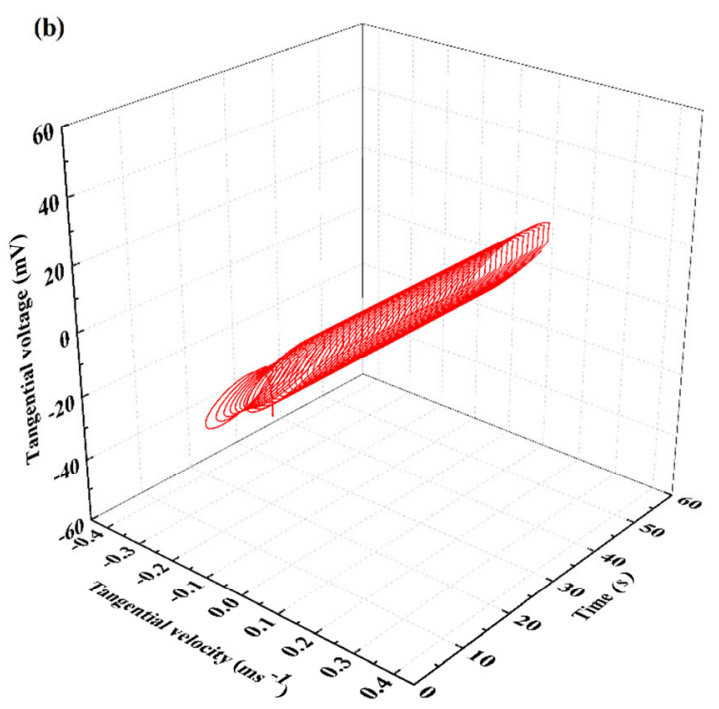

Fig. 15 The 3D phase diagrams of output voltages versus velocities and time of piezoelectric system in two directions.
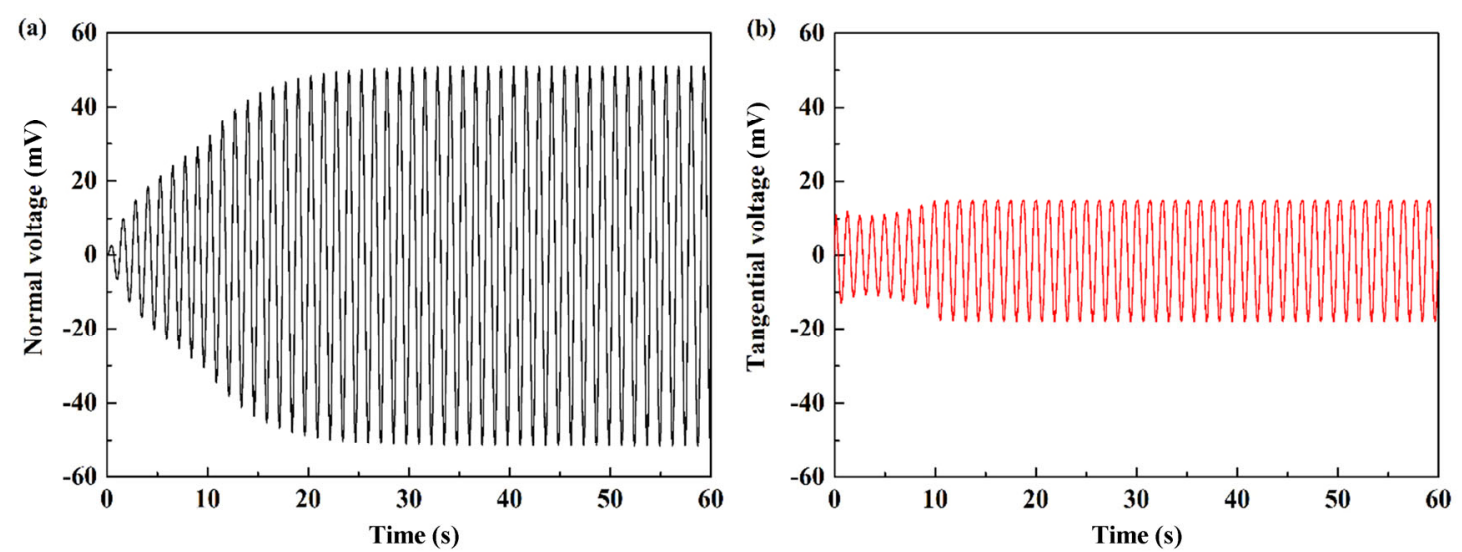

Fig. 16 The time-domain output voltages of piezoelectric system in two directions. 

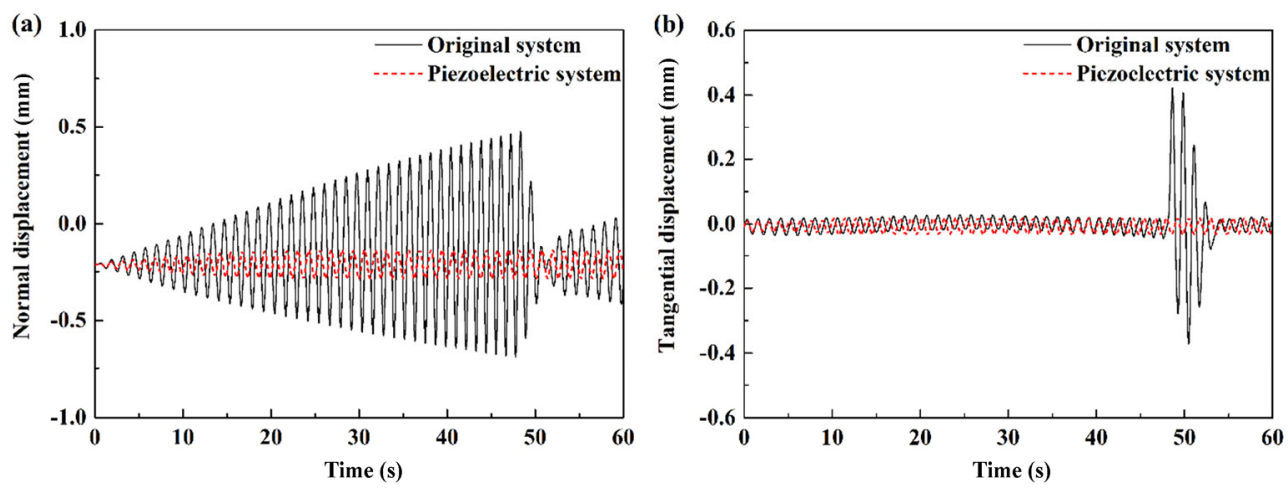

Fig. 17 The time response of displacement of original system and piezoelectric system in two directions.
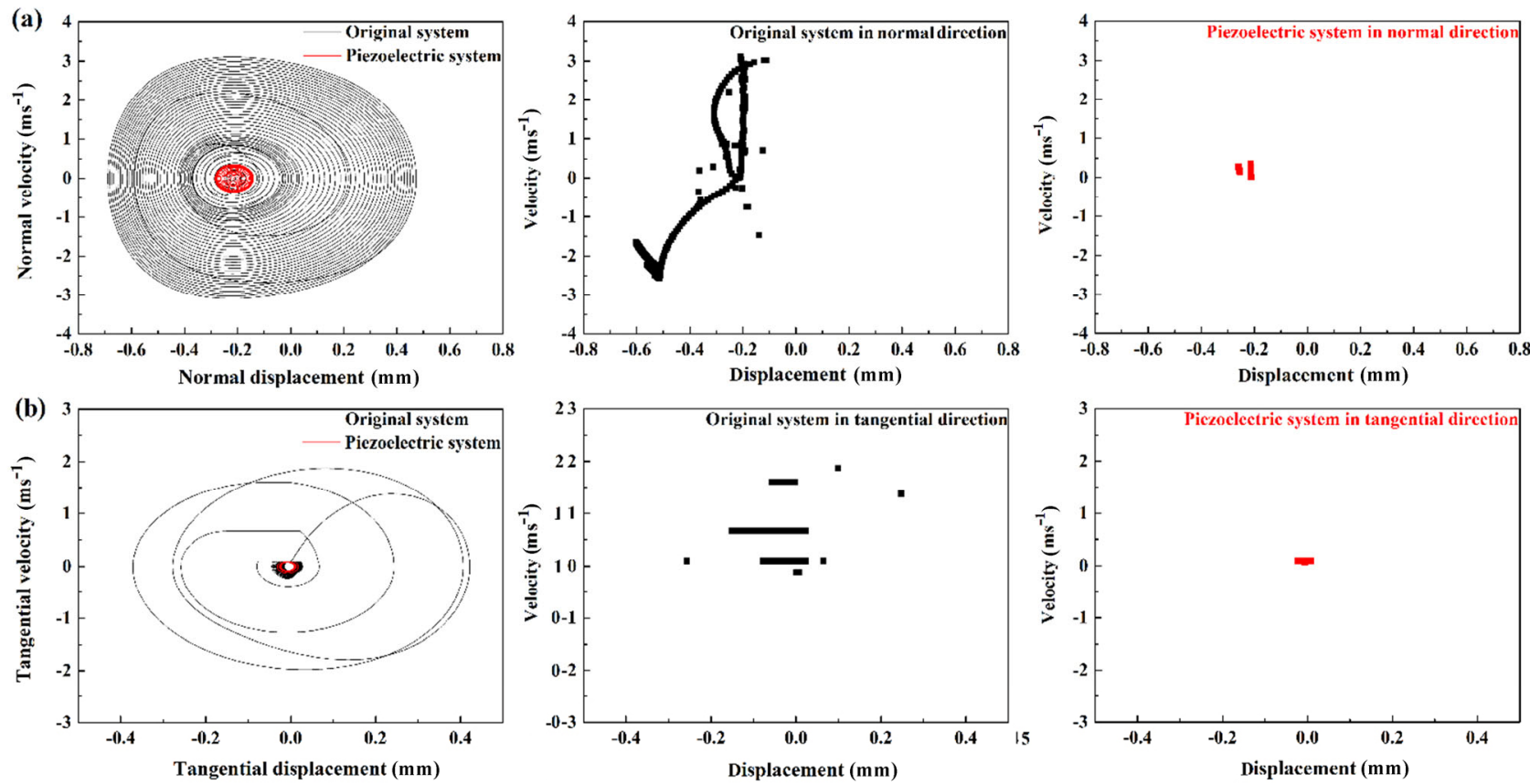

Fig. 18 The velocity and displacement phase and Poincare maps of the original system and piezoelectric system in two directions.

and 18(b) show that the original system model has a large amplitude (limit-cycle) response in both the normal direction and the tangential direction. Under the same system parameters, the steady-state vibration amplitude of the piezoelectric system model is much smaller than that of the original system model in both directions.

It can be clearly seen from the Poincare maps of the original system that a large number of points are displayed in the normal and tangential directions and many of these points are connected together, which indicates that the vibration of the original system in both directions is highly complicated. However, the Poincare maps of the piezoelectric system in the normal and tangential directions display only a few concentrated point clusters, which indicates that the vibration is nearly periodic. These results are in good qualitative agreement with the experimental results shown in Section 3.2.2, indicating that the piezoelectric elements of the piezoelectric system can convert part of the vibration energy into electrical energy, thereby reducing the FIV level of the friction system, which also provides a possible method for reducing the FIV level.

\section{Conclusions}

In this work, a reciprocating friction test device was constructed, which can convert part of the FIV energy into electrical energy via attached piezoelectric elements. 
The original friction system and its two variants (the original system with two attached cantilevers and that with two piezoelectric elements added) were tested and analyzed in numerical simulation. The effects of adding piezoelectric elements on the vibration, noise, and surface wear caused by friction were systematically examined. The main conclusions of this study are as follows:

The correlation between the acceleration and the voltage was studied through a coherence analysis, and the feasibility of converting FIV energy into electrical energy was proven. The experimental results show that adding a piezoelectric element can effectively reduce the amplitude of the vibration acceleration and the sound pressure level of the friction system.

1) Based on the observation of the worn surface morphology by OM, WLI, and SEM, it was found that adding piezoelectric elements into the original friction system has a significant impact on the tribological behavior of the friction system, which can effectively reduce the surface wear.

2) A finite element model of the cantilever system was established, and an implicit dynamic analysis was performed to calculate the displacements of the cantilever beams during the friction process. It is shown that the cantilever beams vibrate during the friction process, and the vibration energy can be transferred into electric energy if piezoelectric elements are added.

3) Two-degree-of-freedom models of the original friction system and the piezoelectric system were established. The numerical simulation results prove not only the feasibility of converting FIV energy into electric energy, but also the reduction of FIV of the piezoelectric elements.

This work provides a new method for reducing surface wear and inhibiting or eliminating FIVN. By converting part of the FIV energy into electrical energy, the performance of the interface friction vibration noise is improved, the generation of FIVN can be effectively inhibited and surface wear can be reduced. However, this work is still a fundamental study about the effect of piezoelectric energy harvesting on the FIVN performance and wear behavior of friction systems. The harvested energy is considerably small and is not usable in practical cases since rectifying circuit is not designed. And the converted vibration energy is also reduced when the FIV is suppressed, which lowers the energy conversion efficiency. Future work is going to focus on the improvement of the energy conversion efficiency and the rectifying circuit to meet the practical application of FIV energy harvesting.

\section{Acknowledgements}

Xiaofan Wang wishes to thank Dr. Zilin Li of Zhengzhou University, Mr. Bo Huang, and Miss Runlan Wang of Southwest Jiaotong University for their help in coding and computing work done in this paper. This project was supported by the National Natural Science Foundation of China (Nos. 51822508 and 11672052) and the Sichuan Province Science and Technology Support Program (No. 2020JDTD0012).

Open Access This article is licensed under a Creative Commons Attribution 4.0 International License, which permits use, sharing, adaptation, distribution and reproduction in any medium or format, as long as you give appropriate credit to the original author(s) and the source, provide a link to the Creative Commons licence, and indicate if changes were made.

The images or other third party material in this article are included in the article's Creative Commons licence, unless indicated otherwise in a credit line to the material. If material is not included in the article's Creative Commons licence and your intended use is not permitted by statutory regulation or exceeds the permitted use, you will need to obtain permission directly from the copyright holder.

To view a copy of this licence, visit http://creativecommons.org/licenses/by/4.0/.

\section{References}

[1] Beeby S P, Tudor M J, White N M. Energy harvesting vibration sources for microsystems applications. Meas Sci Technol 17: 175-195 (2006)

[2] $\mathrm{Fu} \mathrm{H}$, Yeatman E M. A methodology for low-speed broadband rotational energy harvesting using piezoelectric transduction and frequency up-conversion. Energy 125(15): 152-161 (2017)

[3] He J, Wen T, Qian S, Zhan Z X, Tian Z M, Zhu J, Mu J L, Hou X J, Geng WP, Han J D, et al. Triboelectricpiezoelectric-electromagnetic hybrid nanogenerator for high- 
efficient vibration energy harvesting and self-powered wireless monitoring system. Nano Energy 43: 326-339 (2018)

[4] Liu J Q, Fang H B, Xu Z Y, Mao X H, Shen X C, Chen D, Liao H, Cai B C. A MEMS-based piezoelectric power generator array for vibration. Microelectron J 39(5): 802-806 (2013)

[5] Jeon Y B, Sood R, Jeong J H, Kim S G. MEMS power generator with transverse mode thin film PZT. Sens Actuators A 122(1): 16-22 (2005)

[6] Iiyas M A, Swingler J. Piezoelectric energy harvesting from raindrop impacts. Energy 90(1): 796-806 (2015)

[7] Tao K, Wu J, Tang L H, Xia X, Lye S W, Miao J M, Hu X. A novel two-degree-of-freedom MEMS electromagnetic vibration energy harvester. $J$ Micromech Microeng 26: 035020 (2016)

[8] Xie X D, Wang Q. Energy harvesting from a vehicle suspension system. Energy 86(15): 385-392 (2015)

[9] Zhang Y L, Wang T Y, Luo A X, Hu Y S, Li X X, Wang F. Micro electrostatic energy harvester with both broad bandwidth and high-normalized power density. Appl Energy 212(15): 362-371 (2018)

[10] Fu Y Q, Ouyang H, Davis R B. Nonlinear dynamics and triboelectric energy harvesting from a three-degree-of-freedom vibro-impact oscillator. Nonlinear Dyn 92(4): 1985-2004 (2018)

[11] Fang H B, Liu J Q, Xu Z Y, Dong L, Wang L, Che D, Cai B C, Liu Y. Fabrication and performance of MEMS-based piezoelectric power generator for vibration energy harvesting. Microelectron J 37(11): 1280-1284 (2018)

[12] Erturk A, Inman D J. A distributed parameter electromechanical model for cantilevered piezoelectric energy harvesters. $J$ Vib Acoust 130(4): 041002-041016 (2008)

[13] Sodano H A, Inman D J, Park G. Comparison of piezoelectric energy harvesting devices for recharging batteries. J Intell Mater Syst Struct 16(10): 799-807 (2005)

[14] Yang Y W, Tang L H. Equivalent circuit modeling of piezoelectric energy harvesters. J Intell Mater Syst Struct 20(18): 2223-2235 (2009)

[15] Saadon S, Sidek O. A review of vibration-based MEMS piezoelectric energy harvesters. Energy Convers Manage 52(1): 500-544 (2011)

[16] Kim H S, Kim J H, Kim J. A review of piezoelectric energy harvesting based on vibration. Int J Precis Eng Manuf 12(6): 1129-1141 (2011)

[17] Erturk A, Inman D J. In Piezoelectric energy harvesting. Eds. Chichester: John Wiley \& Sons, 2011: 1-18.

[18] Wei C F, Jing X J. A comprehensive review on vibration energy harvesting: modelling and realization. Renew Sust Energ Rev 74: 1-18 (2017)

[19] Stewart M, Weaver P M, Cain M. Charge redistribution in piezoelectric energy harvesters. Appl Phys Lett 100(7): 073901-073903 (2012)

[20] Yang Z B, Zhou S X, Inman D J. High-performance piezoelectric energy harvesters and their applications. Joule 2(4): 642-697 (2018)

[21] Tadokoro C, Matsumoto A, Nagamine T, Sasaki S. Piezoelectric power generation using friction-induced vibration. Smart Mater Stru 26: 065012 (2017)

[22] Kang J, Kim K. Squeak noise in lead screw systems: selfexcited vibration of continuous model. J Sound Vibr 329(17): 3587-3595 (2010)

[23] Chatterjee S. Non-linear control of friction-induced self-excited vibration. Int J Non-Linear Mech 42(3): 459-469 (2007)

[24] Hu W P, Wang P G, Chen X, Hu Y, Cui X L, Peng J F, Zhu $\mathrm{M}$ H. Experimental study on corrugation of a sliding surface caused by frictional self-excited vibration. Tribol Trans 59(1): 8-16 (2016)

[25] Chen G X, Zhou Z R. Time-frequency analysis of frictioninduced vibration under reciprocating sliding conditions. Wear 262(1-2): 1-10 (2007)

[26] Yu H L, Walsh S J, Chen G, Zhang L, Qian K, Wang L. Analysis of friction-induced vibration leading to brake squeal using a three degree-of-freedom model. Tribol Lett 65(105): 1-13 (2017)

[27] Ghazaly N M, El-Sharkawy M, Ahmed I. A review of automotive brake squeal mechanisms. J Mech Des Vib 1(1): 5-9 (2013)

[28] Wang D W, Mo J L, Ouyang H, Zhou Z R. Improving dynamic and tribological behaviours by means of a $\mathrm{Mn}-\mathrm{Cu}$ damping alloy with grooved surface features. Tribol Lett 66(67): 1-16 (2018)

[29] Wang D W, Mo J L, Wang Z G, Chen G X, Ouyang H, Zhou Z R. Numerical study of friction-induced vibration and noise on groove-textured surface. Tribol Int 64: 1-7 (2013)

[30] Bot A L, Chakra E B. Measurement of friction noise versus contact area of rough surfaces weakly loaded. Tribol Lett 37: 273-281 (2010)

[31] Liu T, Li G B, Wei H J, Sun D. Experimental observation of cross correlation between tangential friction vibration and normal friction vibration in a running-in process. Tribol Int 97: 77-88 (2016)

[32] Rouzic J Le, Bot A L, Perret-Liaudet J, Guibert M, Rusanov A, Douminge L, Bretagnol F, Mazuyer D. Friction-induced vibration by Stribeck's law: Application to wiper blade squeal noise. Tribol Lett 49(3): 563-572 (2013)

[33] Wu H, Tang L H, Yang Y W, Soh C K. A novel twodegrees-of-freedom piezoelectric energy harvester. $J$ Intell Mater Syst Struct 24(3): 357-368 (2013)

[34] Sinou J J, Cayer-Barrioz J, Berro H. Friction-induced 
vibration of a lubricated mechanical system. Tribol Int $\mathbf{6 1}$ : 156-168 (2013)

[35] Kim S S, Hwang H J, Shin M W, Jang H. Friction and vibration of automotive brake pads containing different abrasive particles. Wear 271(7-8): 1194-1202 (2011)

[36] Tsujiura Y, Suwa E, Nishi T, Kurokawa F, Hida H, Kanno I. Airflow energy harvester of piezoelectric thin-film bimorph using self-excited vibration. Sens Actuator B-Chem 261: 295-301 (2017)

[37] Tadokoro C, Matsumoto A, Nagamine T, Sasaki S. Piezoelectric power generation using friction-induced vibration. Smart Mater Stru 26: 065012 (2017)

[38] Helseth, L E. Excitation of energy harvesters using stick-slip motion. Smart Mater Stru 23: 085024 (2014)

[39] Tadokoro C, Matsumoto A, Nagamine T, Sasaki S. Piezoelectric power generation using friction-induced vibration. Smart Mater Stru 26: 065012 (2017)

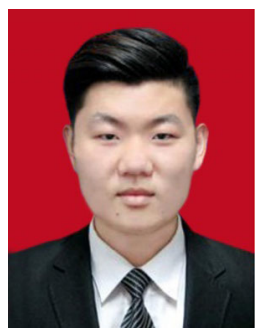

Xiaofan WANG. He received his bachelor degree in mechanical engineering in 2017 from Dalian Jiaotong University, Dalian, China. After then, he received his master

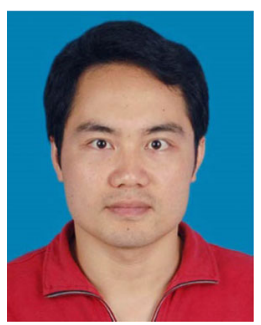

Jiliang MO. He received his M.S. and Ph.D. degrees in in mechanical design and theory from Southwest Jiaotong University, Chendu, China, in 2003 and 2008, respectively. From 2011 to 2012, he worked as a

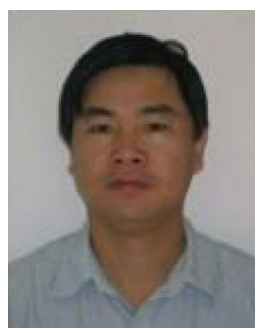

Huajiang OUYANG. He received his Ph.D. degree in structural engineering in 1985 from Dalian University of Technology, Dalian, China. He was an associate professor in the Department of Engineering Mechanics at Dalian University of
[40] Masuda A, Sawai C. Stick-slip energy harvesting: A preliminary study. Smart Mater Adapt Stru Intell Syst 1: 18-20 (2017)

[41] Wang D W, Mo J L, Wang X F, Ouyang H, Zhou Z R. Experimental and numerical investigations of the piezoelectric energy harvesting via friction-induced vibration. Energy Convers Manage 171: 1134-1149 (2018)

[42] Wang D W, Mo J L, Zhu Z Y, Ouyang H, Zhu M H, Zhou $Z$ R. Debris trapping and space-varying contact via surface texturing for enhanced noise performance. Wear 396-397(15): 86-97 (2018)

[43] Li Z L, Ouyang H, Guan Z Q. Nonlinear friction-induced vibration of a slider-belt system. $J$ Vib Acoust 138(4): 1006-1009 (2016)

[44] Lefeuvre E, Badel A, Richard C, Petit L, Guyomar D. A comparison between several vibration-powered piezoelectric generators for standalone systems. Sens Actuator A-Phys 126(2): 405-416 (2006)

degree in mechanical engineering in 2020 from Southwest Jiaotong University, Chendu, China. His research interests include tribology and energy harvesting based on friction-induced vibration.

visiting scholar at University of Sheffield in UK. He has become a professor of the School of Mechanical Engineering in Southwest Jiaotong University since 2016. His research interests include tribology, surface treatment, and dynamic finite element analysis.

Technology (1985-1993). After that, he became a professor of the School of Engineering in the University of Liverpool. He is currently a professor and school director of Postgraduate Research at University of Liverpool. His research interests include mechanical properties, numerical modeling, and friction-induced vibration. 


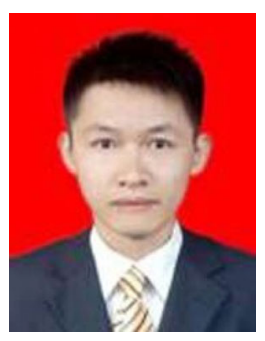

Zaiyu XIANG. He received his M.S. degree in mechanical engineering in 2016 from Kunming University of Science and Technology, Kunming, China. After then, he was a lecturer in the Faculty of Mechanical and

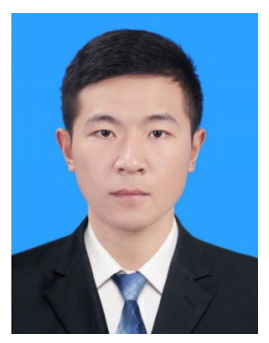

Wei CHEN. He received his bachelor degree in mechanical engineering in 2018 from Southwest Jiaotong University, Chengdu, China. Currently, he is a master candidate in

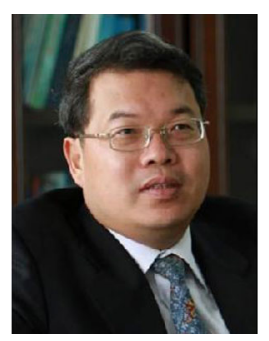

Zhongrong ZHOU. He received his Ph.D. from Ecole Centrale De Lyon, France. After that, he took his postdoctoral research in Laval University, Canada. From 1995 to 1997, he was invited to serve as the guest professor in Ecole Centrale
Electrical Engineering, Liuzhou Vocational and Technical College, Liuzhou, China. Currently, he is a Ph.D. candidate in the School of Mechanical Engineering, Southwest Jiaotong University, Chengdu, China. His research interests include friction-induced vibration and energy harvesting.

the Department of Mechanical Engineering, Southwest Jiaotong University, Chengdu, China. His research interests include friction-induced vibration (FIV) and energy harvesting via FIV.

De Lyon, France. He has been appointed as a dean of School of Mechanical Engineering in Southwest Jiaotong University since 2006. His current position is a professor and vice-president of Southwest Jiaotong University. His research interests include tribology, surface engineering technology, and materials science. 